\title{
AFFINE-PERIODIC SOLUTIONS FOR NONLINEAR DIFFERENTIAL EQUATIONS
}

\author{
CHUANBIAO WANG, XUE YANG AND YONG LI
}

\begin{abstract}
The existence of affine-periodic solutions is studied. These types of solutions may be periodic, harmonic or even quasi-periodic. Mainly, via the topological degree theory, a general existence theorem is proved, which asserts the existence of affine-periodic solutions, extending some classical results. The theorem is applied to establish the Lyapunov function type theorem and the invariant region principle relative to affine-periodic solutions.
\end{abstract}

1. Introduction. Great Newton discovered the law of universal gravitation by applying his calculus, and firstly proved Kapler's laws of planetary motion mathematically, in which he showed that the orbit of planetary motion is elliptical. This was the first result on the existence of periodic solutions for differential equations. At the end of the 19th century, in the study of celestial mechanics, Poincaré considered periodic solutions and developed a theory which can show properties such as periodicity by discussing the characteristics of equations without solving them. This theory is called the qualitative theory of differential equations together with the work of Lyapunov on the stability of solutions. The Poincaré map and the Lyapunov function are still very important instruments in the research of periodic solutions.

Periodicity is a very important property in the study of differential equations, but not all natural phenomena can be described alone by periodicity. In fact, some differential equations often exhibit certain

2010 AMS Mathematics subject classification. Primary 34C25, 34C27, 47H11.

Keywords and phrases. Affine-periodic solution, Lyapunov function, topological degree, invariant region principle.

This work was supported by the National Basic Research Program of China (grant No. 2013CB834100) and the National Natural Science Foundation of China (grant Nos. 11171132, 11201173, 11571065). The second author is the corresponding author. 2014.

Received by the editors on July 27, 2014, and in revised form on December 16, 
symmetries rather than periodicity. For example, consider the system:

$$
x^{\prime}=f(t, x),
$$

where $f(t, x): R^{1} \times R^{n} \rightarrow R^{n} ; x^{\prime}$ stands for

$$
\frac{\mathrm{d} x}{\mathrm{~d} t},
$$

and, for some $Q \in \operatorname{GL}\left(R^{n}\right)$, the following affine symmetry holds:

$$
f(t+T, x)=Q f\left(t, Q^{-1} x\right) .
$$

In the sense of (1.2), we have the concept of the affine-periodic system (APS).

Definition 1.1. Consider system (1.1). If there exists $Q \in \mathrm{GL}(n)$ and $T>0$ such that

$$
f(t+T, x)=Q f\left(t, Q^{-1} x\right)
$$

holds for all $(t, x) \in R^{1} \times R^{n}$, we call (1.1) a $(Q, T)$-affine-periodic system.

Remark 1.2. Consider system (1.1). If there is a linear transformation of coordinates $B$ which makes $y=B x$, then

$$
\frac{\mathrm{d} y}{\mathrm{~d} t}=\frac{\mathrm{d} B x}{\mathrm{~d} t}=B f\left(t, B^{-1} y\right) .
$$

Letting $g(t, y)=B f\left(t, B^{-1} y\right)$, we have

$$
g(t+T, y)=B f\left(t+T, B^{-1} y\right)=B Q f\left(t, Q^{-1} B^{-1} y\right),
$$

and

$$
\widehat{Q} g\left(t, \widehat{Q}^{-1} y\right)=\widehat{Q} B f\left(t, B^{-1} \widehat{Q}^{-1} y\right)=B Q f\left(t, Q^{-1} B^{-1} y\right),
$$

where $\widehat{Q}=B Q B^{-1}$. Hence,

$$
g(t+T, y)=\widehat{Q} g\left(t, \widehat{Q}^{-1} y\right) .
$$

This means that linear transformations of coordinates keep the affineperiodicity of system (1.1). Obviously, for general nonlinear transformation of coordinates such as $y=h(x)$, the affine-periodicity will no longer hold. 
By Definition 1.1, it is easy to see that this affine-periodic invariance exhibits two characters: periodicity in time and symmetry in space. Obviously, when $Q=$ id (identity matrix), the invariance is just the pure periodicity; when $Q=-\mathrm{id}$, the invariance implies the usual antisymmetry in space; when $Q \in O(n)$, the invariance shows rotating symmetry in space. For some relative studies, we refer to $[\mathbf{1}, \mathbf{2}, \mathbf{3}]$.

Now a basic topic is to investigate the existence of $(Q, T)$-affineperiodic solutions $x(t)$ of system (1.1), i.e.,

$$
x(t+T)=Q x(t) .
$$

In fact, this problem is equivalent to proving the existence of solutions of the BVP in the following lemma.

Lemma 1.3. Consider system (1.1), where $f(t, x)$ is continuous and ensures the uniqueness of solutions with respect to initial values. The existence of $(Q, T)$-affine-periodic solutions of equation (1.1) is equivalent to the existence of solutions of the BVP (1.1) with $x(T)=Q x(0)$.

Proof. For any solution $x(t)$ of equation (1.1), let $u(t)=Q^{-1} x(t+T)$. Then:

$$
\begin{aligned}
\frac{\mathrm{d} u(t)}{\mathrm{d} t} & =\frac{Q^{-1} \mathrm{~d} x(t+T)}{\mathrm{d}(t+T)}=Q^{-1} f(t+T, x(t+T)) \\
& =Q^{-1}\left(Q f\left(t, Q^{-1} x(t+T)\right)\right)=f(t, u(t)) .
\end{aligned}
$$

This shows that $u(t)$ is a solution of equation (1.1). By the uniqueness of solutions with respect to initial values and $u(0)=Q^{-1} x(T)$, we know that $u(t)=Q^{-1} x(t+T) \equiv x(t)$ if and only if $Q^{-1} x(T)=x(0)$.

As a structural property of functions, affine periodicity is a generalization of pure periodicity. Some results have been proved to be similar to periodic systems. For example, Yoshizawa proved that each dissipative periodic system admits a periodic solution [13]. For dissipative affine-periodic systems, the existence of affine-periodic solutions had been proved [14]. In addition, Li, et al., discussed Levinson's problem on affine-periodic solutions where the system is dissipative-repulsive [8], while some similar results on periodic solutions can be found in $[4,5,6,12]$, and the problem was finally solved by Küpper et al. [7]. 
In this paper, we are interested in the existence of affine-periodic solutions of systems without those conditions such as dissipativeness. With the use of topological degree theory, some existence theorems are proven for affine-periodic systems with $Q \in O(n)$. By using these theorems, different conditions of Lyapunov function are given which can be used to study the existence of affine-periodic solutions. We also prove an invariant region principle on the existence of affine-periodic solutions. Although this kind of principle has become popular in the study of periodic solutions, it is unknown wether similar results hold in quasi-periodic or almost periodic solutions. Now, our affine-periodic solutions might be quasi-periodic.

Let us state our main results as follows.

Consider APS (1.1)

$$
x^{\prime}=f(t, x),
$$

where the $(Q, T)$-affine-periodic function $f: R^{1} \times R^{n} \rightarrow R^{n}$ is continuous and ensures the uniqueness of solutions with respect to initial values, $Q \in O(n)$.

To investigate the existence of solutions of equation (1.1), one often considers the following auxiliary equation,

$$
x^{\prime}=\lambda f(t, x),
$$

where $\lambda \in[0,1]$.

We first introduce an existence theorem for affine-periodic solutions. The proof can be found in Section 2 .

Theorem 1.4. Let $D \subset R^{n}$ be a bounded open set. Assume the following hypotheses hold for equation $(1)_{\lambda}$.

$\left(H_{1}\right)$ For each $\lambda \in(0,1]$, every possible affine-periodic solution $x(t)$ of equation $(1)_{\lambda}$ satisfies

$$
x(t) \notin \partial D \text { for all } t
$$

$\left(\mathrm{H}_{2}\right)$ the Brouwer degree,

$$
\operatorname{deg}(g, D \cap \operatorname{Ker}(I-Q), 0) \neq 0, \quad \text { if } \operatorname{Ker}(I-Q) \neq\{0\},
$$


where

$$
g(a)=\frac{1}{T} \int_{0}^{T} \operatorname{Pf}(s, a) \mathrm{d} s,
$$

$P: R^{n} \rightarrow \operatorname{Ker}(I-Q)$ is an orthogonal projection. Then equation (1.1) has at least one $(Q, T)$-affine-periodic solution $x_{*}(t) \in D$ for all $t$.

Remark 1.5. When $Q=\mathrm{id}$, APS (1.1) is just the usual periodic system and $\operatorname{Ker}(I-Q)=R^{n}$. In this situation, our result is the same as Mawhin's famous theorem [10].

Remark 1.6. When $\operatorname{Ker}(I-Q)=\{0\}$, if we can make the prior estimate in $\left(H_{1}\right)$, then we can conclude the existence of $(Q, T)$-affineperiodic solutions. In this situation, the solutions are just special quasiperiodic ones. It is well known that the study of quasi-periodic solutions is rather difficult due to the "small divisor problem." The celebrated KAM theory [9] provides a powerful tool in solving the persistence of quasi-periodic solutions from the formulism of perturbation. Theorem 1.4 asserts the existence of some special quasi-periodic solutions in certain topological formalism.

Theorem 1.4 offers a topological method for studying the existence of affine-periodic solutions. When dealing with specific problems, we hope to have a more direct method. The Lyapunov function method is a very useful instrument in the study of solutions. Here, we give two results on the basis of Lyapunov functions. The first one has been proved by Wang, et al., in [11]. We give a new proof in this paper, and the second one is new.

Corollary 1.7. Consider equation (1.1), where the affine-periodic function $f: R^{1} \times R^{n} \rightarrow R^{n}$ is continuous and ensures the uniqueness of solutions with respect to initial values. Assume that there exist $C^{1}$ functions $V_{i}(x), i=0,1, \ldots, m$ and $\sigma>0$, such that the following hold.

$\left(\mathrm{H}_{3}\right)$ For $M_{i}$ large enough,

$$
\left|\left\langle\nabla V_{i}(x), f(t, x)\right\rangle\right| \geq \sigma>0 \text { for all }|x| \geq M_{i}, i=0,1, \ldots, m, t \in R^{1} .
$$

And, if $\operatorname{Ker}(I-Q) \neq\{0\}$,

$$
\left|\left\langle\nabla V_{i}(x), \operatorname{Pf}(t, x)\right\rangle\right| \geq \sigma>0
$$


for all $x \in \operatorname{Ker}(I-Q)$ and $|x| \geq M_{i}, i=0,1, \ldots, m, t \in R^{1}$, where $P: R^{n} \rightarrow \operatorname{Ker}(I-Q)$ is an orthogonal projection;

$\left(H_{4}\right)$

$$
\sum_{i=0}^{m}\left|V_{i}(x)\right| \longrightarrow \infty, \quad \text { as }|x| \rightarrow \infty
$$

$\left(\mathrm{H}_{5}\right)$ the Brouwer degree,

$$
\operatorname{deg}\left(\nabla V_{0}, B_{M_{0}} \cap \operatorname{Ker}(I-Q), 0\right) \neq 0, \quad \text { if } \operatorname{Ker}(I-Q) \neq\{0\},
$$

where $B_{\rho}=\left\{p \in R^{n}:|p|<\rho\right\}$. Then equation (1.1) has at least one $(Q, T)$-affine-periodic solution $x_{*}(t)$.

As a more concrete application of Corollary 1.7, we obtain the following.

Corollary 1.8. Consider system (1.1). Assume that there exists a constant $M>0$, such that

$$
|\langle x, f(t, x)\rangle| \geq \sigma>0 \quad \text { for all }|x| \geq M, t \in R^{1},
$$

and if $\operatorname{Ker}(I-Q) \neq\{0\}$, $|\langle x, \operatorname{Pf}(t, x)\rangle| \geq \sigma>0 \quad$ for all $x \in \operatorname{Ker}(I-Q)$ and $|x| \geq M, t \in R^{1}$, where $P: R^{n} \rightarrow \operatorname{Ker}(I-Q)$ is an orthogonal projection. Then equation (1.1) has at least one $(Q, T)$-affine-periodic solution $x_{*}(t)$.

Proofs of Corollaries 1.7 and 1.8 can be found in Section 3.

The following result shows how Lyapunov functions work on the boundary of the domain.

Theorem 1.9. Consider equation (1.1), where the affine-periodic function $f: R^{1} \times R^{n} \rightarrow R^{n}$ is continuous and ensures the uniqueness of solutions with respect to initial values. Let $V: \bar{D} \rightarrow R$ be a $C^{1}$ function such that the following hold.

$\left(H_{6}\right) D$ is a bounded open set;

$\left(H_{7}\right)$ there exists a constant $\sigma>0$ such that

$$
|\langle\nabla V(x), f(t, x)\rangle| \geq \sigma \quad \text { for all }(t, x) \in R^{1} \times \partial D,
$$


and, if $\operatorname{Ker}(I-Q) \neq\{0\}$,

$$
|\langle\nabla V(x), P f(t, x)\rangle| \geq \sigma \quad \text { for all }(t, x) \in R^{1} \times \partial(D \cap \operatorname{Ker}(I-Q)),
$$

where $P: R^{n} \rightarrow \operatorname{Ker}(I-Q)$ is an orthogonal projection;

$\left(H_{8}\right)$

$$
\operatorname{deg}(\nabla V, D \cap \operatorname{Ker}(I-Q), 0) \neq 0, \quad \text { if } \operatorname{Ker}(I-Q) \neq\{0\} .
$$

Then equation (1.1) has at least one $(Q, T)$-affine-periodic solution $x_{*}(t) \in D$ for all $t$.

The proof can be found in Section 4.

When equation (1.1) is $T$-periodic, we know that if all the solutions of (1.1) are inward to $D$ from the boundary $\partial D$, then (1.1) admits a $T$-periodic solution. This result is known as the invariant region principle. Does a similar result hold for affine-periodic solutions? The answer is positive. The following theorem gives us an affine-periodic type invariant region principle.

Before giving the last theorem, we first introduce the definition of the hull.

Definition 1.10. Let $T$ denote the translation operator, and let $\alpha$ stand for a sequence $\left\{\alpha_{n}\right\} . T_{\alpha} f=l$ means that

$$
l(t)=\lim _{n \rightarrow \infty} f\left(t+\alpha_{n}\right)
$$

and is only written when the limit exists. Then,

$$
H(f) \doteq\left\{l: \text { there exists } \alpha \text { with } T_{\alpha} f=l \text { uniformly }\right\}
$$

is called the hull of $f$.

Theorem 1.11. Consider equation (1.1), where $f: R^{1} \times R^{n} \rightarrow R^{n}$ is continuous and $(Q, T)$-affine-periodic. Let $D \subset R^{n}$ be a bounded open and simply connected set, such that $\partial D$ is piecewisely smooth. Let $H(f)$ denote the hull of $f$. Assume the following hold.

$\left(H_{9}\right)$ For every $(t, p) \in R^{1} \times \partial D$ and $h \in H(f), h(t, p)$ is inward toward D; 
$\left(H_{10}\right) g(a) \neq 0$ for all $a \in \partial D$, where

$$
g(a)=\frac{1}{T} \int_{0}^{T} \operatorname{Pf}(s, a) \mathrm{d} s,
$$

$P: R^{n} \rightarrow \operatorname{Ker}(I-Q)$ is an orthogonal projection. Then equation (1.1) has at least one $(Q, T)$-affine-periodic solution $x_{*}(t) \in D$ for all $t$.

This theorem is proven in Section 5.

2. Proof of Theorem 1. To prove this theorem, we introduce the definition of a retraction map.

Definition 2.1. Let $X$ be a topological space and $A$ a subspace of $X$. Then a continuous map $r: X \rightarrow A$ is called a retraction if the restriction of $r$ to $A$ is the identity map on $A$.

Now, we can prove Theorem 1.4.

Proof. Consider the auxiliary equation

$$
x^{\prime}=\lambda f(t, x)
$$

with boundary value condition $x(T)=Q x(0)$, where $\lambda \in[0,1]$. Let $x(t)$ be any solution of $(1)_{\lambda}$ with $x(T)=Q x(0)$. Rewriting $(1)_{\lambda}$ in the form of an equivalent integral equation, we obtain

$$
x(0)+\lambda \int_{0}^{T} f(\tau, x(\tau)) \mathrm{d} \tau=Q x(0) .
$$

Denote $x(0)$ by $x_{0}$. Then

$$
(I-Q) x_{0}=-\lambda \int_{0}^{T} f(\tau, x(\tau)) \mathrm{d} \tau,
$$

where $I$ is the identity matrix.

Consider (2.1) in two parts.

(I) If $\operatorname{Ker}(I-Q) \neq\{0\}$. In this case, $(I-Q)^{-1}$ does not exist. By coordinate transformation, we can just let

$$
Q=\left(\begin{array}{cc}
I & 0 \\
0 & Q_{1}
\end{array}\right)
$$


without loss of generality, where $\left(I-Q_{1}\right)^{-1}$ exists.

Let $P: R^{n} \rightarrow \operatorname{Ker}(I-Q)$ be the orthogonal projection. Then:

$$
\begin{aligned}
(I-Q) x_{0} & =(I-Q)\left(x_{\mathrm{ker}}^{0}+x_{\perp}^{0}\right) \\
& =-\lambda \int_{0}^{T} f(\tau, x(\tau)) \mathrm{d} \tau \\
& =-\lambda \int_{0}^{T} P f(\tau, x(\tau)) \mathrm{d} \tau-\lambda \int_{0}^{T}(I-P) f(\tau, x(\tau)) \mathrm{d} \tau,
\end{aligned}
$$

where $x_{\mathrm{ker}}^{0} \in \operatorname{Ker}(I-Q), x_{\perp}^{0} \in \operatorname{Im}(I-Q)$ and $x_{0}=x_{\mathrm{ker}}^{0}+x_{\perp}^{0}$.

Let $L_{p}=\left.(I-Q)\right|_{\operatorname{Im}(I-Q)}$. It is easy to see that $L_{p}^{-1}$ exists. Thus, equation $(2.2)$ is equivalent to:

$$
\begin{aligned}
& (I-Q) x_{\mathrm{ker}}^{0}=-\lambda \int_{0}^{T} P f(\tau, x(\tau)) \mathrm{d} \tau=0, \\
& (I-Q) x_{\perp}^{0}=-\lambda \int_{0}^{T}(I-P) f(\tau, x(\tau)) \mathrm{d} \tau .
\end{aligned}
$$

Thus, we have

$$
x_{\perp}^{0}=-\lambda L_{p}^{-1}(I-P) \int_{0}^{T} f(\tau, x(\tau)) \mathrm{d} \tau .
$$

Let

$$
X=\left\{x:[0, T] \rightarrow R^{n}: x(t) \text { is continuous on }[0, T]\right\}
$$

and define the norm as $\|x\|=\sup _{t \in[0, T]}|x(t)|$. It is easy to see that $X$ is a Banach space with the norm $\|\cdot\|$. 
For $x \in X$ which satisfies that $x(t) \in \bar{D}$ for all $t \in[0, T]$, we define an operator $T\left(x_{\mathrm{ker}}^{0}, x, \lambda\right)$ by

$$
\begin{aligned}
& T\left(x_{\mathrm{ker}}^{0}, x, \lambda\right)(t) \\
= & \left(\begin{array}{c}
x_{\mathrm{ker}}^{0}+(1 / T) \int_{0}^{T} P f(\tau, x(\tau)) \mathrm{d} \tau \\
x_{\mathrm{ker}}^{0}-\lambda L_{p}^{-1}(I-P) \int_{0}^{T} f(\tau, x(\tau)) \mathrm{d} \tau+\lambda \int_{0}^{t} f(\tau, x(\tau)) \mathrm{d} \tau,
\end{array}\right),
\end{aligned}
$$

where $\lambda \in[0,1]$. We claim that each fixed point $x$ of $T$ in $X$ is a solution of $(1)_{\lambda}$ with $x(T)=Q x(0)$.

In fact, if $x$ is a fixed point of $T$, we have

$$
\left(\begin{array}{c}
x_{\mathrm{ker}}^{0} \\
x(t)
\end{array}\right)=\left(\begin{array}{c}
x_{\mathrm{ker}}^{0}+(1 / T) \int_{0}^{T} \operatorname{Pf}(\tau, x(\tau)) \mathrm{d} \tau \\
x_{\mathrm{ker}}^{0}-\lambda L_{p}^{-1}(I-P) \int_{0}^{T} f(\tau, x(\tau)) \mathrm{d} \tau+\lambda \int_{0}^{t} f(\tau, x(\tau)) \mathrm{d} \tau
\end{array}\right) .
$$

Thus,

$$
\frac{1}{T} \int_{0}^{T} \operatorname{Pf}(\tau, x(\tau)) \mathrm{d} \tau=0
$$

$$
x(t)=x_{\mathrm{ker}}^{0}-\lambda L_{p}^{-1}(I-P) \int_{0}^{T} f(\tau, x(\tau)) \mathrm{d} \tau+\lambda \int_{0}^{t} f(\tau, x(\tau)) \mathrm{d} \tau .
$$

By equation (2.5), we know that

$$
x_{0}=x_{\mathrm{ker}}^{0}-\lambda L_{p}^{-1}(I-P) \int_{0}^{T} f(\tau, x(\tau)) \mathrm{d} \tau .
$$

Thus,

$$
\begin{aligned}
Q x_{0} & =Q x_{\mathrm{ker}}^{0}-\lambda Q L_{p}^{-1}(I-P) \int_{0}^{T} f(\tau, x(\tau)) \mathrm{d} \tau \\
& =x_{\mathrm{ker}}^{0}-\lambda Q L_{p}^{-1}(I-P) \int_{0}^{T} f(\tau, x(\tau)) \mathrm{d} \tau .
\end{aligned}
$$

Since equation (2.4) holds, we have

$$
\begin{gathered}
(I-Q) L_{p}^{-1}(I-P) \int_{0}^{T} f(\tau, x(\tau)) \mathrm{d} \tau=(I-P) \int_{0}^{T} f(\tau, x(\tau)) \mathrm{d} \tau \\
=(I-P) \int_{0}^{T} f(\tau, x(\tau)) \mathrm{d} \tau+P \int_{0}^{T} f(\tau, x(\tau)) \mathrm{d} \tau
\end{gathered}
$$




$$
=\int_{0}^{T} f(\tau, x(\tau)) \mathrm{d} \tau
$$

Thus,

$$
\begin{aligned}
\lambda Q L_{p}^{-1}(I-P) & \int_{0}^{T} f(\tau, x(\tau)) \mathrm{d} \tau \\
& =\lambda L_{p}^{-1}(I-P) \int_{0}^{T} f(\tau, x(\tau)) \mathrm{d} \tau-\lambda \int_{0}^{T} f(\tau, x(\tau)) \mathrm{d} \tau .
\end{aligned}
$$

Then,

$$
Q x_{0}=x_{\mathrm{ker}}^{0}-\lambda Q L_{p}^{-1}(I-P) \int_{0}^{T} f(\tau, x(\tau)) \mathrm{d} \tau
$$

$$
=x_{\mathrm{ker}}^{0}-\lambda L_{p}^{-1}(I-P) \int_{0}^{T} f(\tau, x(\tau)) \mathrm{d} \tau+\lambda \int_{0}^{T} f(\tau, x(\tau)) \mathrm{d} \tau=x(T) .
$$

By equations (2.5) and (2.6), we know that equation (2.1) holds. Thus,

$$
x_{\perp}^{0}=-\lambda L_{p}^{-1}(I-P) \int_{0}^{T} f(\tau, x(\tau)) \mathrm{d} \tau .
$$

Then,

$$
\begin{aligned}
x(t) & =x_{\mathrm{ker}}^{0}-\lambda L_{p}^{-1}(I-P) \int_{0}^{T} f(\tau, x(\tau)) \mathrm{d} \tau+\lambda \int_{0}^{t} f(\tau, x(\tau)) \mathrm{d} \tau \\
& =x_{\mathrm{ker}}^{0}+x_{\perp}^{0}+\lambda \int_{0}^{t} f(\tau, x(\tau)) \mathrm{d} \tau=x_{0}+\lambda \int_{0}^{t} f(\tau, x(\tau)) \mathrm{d} \tau .
\end{aligned}
$$

This means that the fixed point $x$ is a solution of $(1)_{\lambda}$ with $x(T)=$ $Q x(0)$.

Now, we need to prove the existence of the fixed point of $T$. Take a constant $M$ which satisfies that

$$
M>\sup _{\substack{t \in[0, T] \\ x \in \bar{D}}} f(t, x) \mid
$$

and let

$$
X_{\lambda}=\left\{x \in X:\left|\frac{x(t)-x(s)}{t-s}\right| \leqslant \lambda M \quad \text { for all } t \neq s\right\} .
$$


Then, it is easy to make a retraction $\alpha_{\lambda}: X \rightarrow X_{\lambda}$.

Define an operator $\widehat{T}\left(x_{\mathrm{ker}}^{0}, x, \lambda\right)$ by

$$
\widehat{T}\left(x_{\mathrm{ker}}^{0}, x, \lambda\right)(t)=\left(\begin{array}{c}
x_{\mathrm{ker}}^{0}+(1 / T) \int_{0}^{T} P f\left(\tau, \alpha_{\lambda} \circ x(\tau)\right) \mathrm{d} \tau \\
\alpha_{\lambda} \circ x_{\mathrm{ker}}^{0}-\lambda L_{p}^{-1}(I-P) \int_{0}^{T} f\left(\tau, \alpha_{\lambda} \circ x(\tau)\right) \mathrm{d} \tau \\
+\lambda \int_{0}^{t} f\left(\tau, \alpha_{\lambda} \circ x(\tau)\right) \mathrm{d} \tau
\end{array}\right) .
$$

As $P: R^{n} \rightarrow \operatorname{Ker}(I-Q)$, it is easy to see that $(1 / T) \int_{0}^{T} P f(\tau, x(\tau)) \mathrm{d} \tau$ $\in \operatorname{Ker}(I-Q)$. Also, $(1 / T) \int_{0}^{T} \operatorname{Pf}\left(\tau, \alpha_{\lambda} \circ x(\tau)\right) \mathrm{d} \tau \in \operatorname{Ker}(I-Q)$.

Let us consider the homotopy:

$$
\begin{gathered}
H\left(x_{\mathrm{ker}}^{0}, x, \lambda\right)=\widehat{T}\left(x_{\mathrm{ker}}^{0}, x, \lambda\right), \\
\left(x_{\mathrm{ker}}^{0}, x, \lambda\right) \in(D \cap \operatorname{Ker}(I-Q)) \times \widetilde{D} \times[0,1],
\end{gathered}
$$

where $\widetilde{D}=\{x \in X: x(t) \in D$ for all $t \in[0, T]\}$.

We claim that

$$
0 \notin(\mathrm{id}-H)(\partial((D \cap \operatorname{Ker}(I-Q)) \times \widetilde{D}) \times[0,1]) .
$$

Suppose, on the contrary, that there exists $\left(\widehat{x}_{\mathrm{ker}}^{0}, \widehat{x}, \widehat{\lambda}\right) \in \partial((D \cap$ $\operatorname{Ker}(I-Q)) \times \widetilde{D}) \times[0,1]$, such that $(\mathrm{id}-H)\left(\widehat{x}_{\mathrm{ker}}^{0}, \widehat{x}, \widehat{\lambda}\right)=0$. As $\widehat{x}_{\mathrm{ker}}^{0} \in \partial D$ is contradictory to $\left(H_{1}\right)$, and $\partial(D \cap \operatorname{Ker}(I-Q)) \subset \partial D$, we have that $\widehat{x}_{\text {ker }}^{0} \notin \partial(D \cap \operatorname{Ker}(I-Q))$. In other words, $\widehat{x} \in \partial \widetilde{D}$. Then equation $(2.11)$ can be proved as follows.

(i) When $\widehat{\lambda}=0$, by the definition of set $X_{\lambda}$, we have

$$
X_{0}=\left\{x \in X:\left|\frac{x(t)-x(s)}{t-s}\right| \leqslant 0 \quad \text { for all } t \neq s\right\} \text {. }
$$

Hence, $\alpha_{0} \circ x(t) \equiv \alpha_{0} \circ x(0)$ for all $t \in[0, T]$. Since $(\mathrm{id}-H)\left(\widehat{x}_{\mathrm{ker}}^{0}, \widehat{x}, 0\right)$ $=0$, we have

$$
\left(\begin{array}{c}
\widehat{x}_{\mathrm{ker}}^{0} \\
\widehat{x}(t)
\end{array}\right)=\left(\begin{array}{c}
\widehat{x}_{\mathrm{ker}}^{0}+(1 / T) \int_{0}^{T} \operatorname{Pf}\left(\tau, \alpha_{\lambda} \circ \widehat{x}(\tau)\right) \mathrm{d} \tau \\
\alpha_{0} \circ \widehat{x}_{\mathrm{ker}}^{0}
\end{array}\right) .
$$

This means that $\widehat{x}(t) \equiv \widehat{x}(0)$ for all $t \in[0, T]$. Taking $\widehat{x}(t)=p$, we have 
$\alpha_{0} \circ \widehat{x}_{\mathrm{ker}}^{0}=\widehat{x}(t)=p$. Consequently,

$$
\frac{1}{T} \int_{0}^{T} \operatorname{Pf}(\tau, p) \mathrm{d} \tau=0
$$

and this is equivalent to $g(p)=0$ by the definition of $g(a)$. Notice that $\widehat{x} \in \partial \widetilde{D}$ and $\widetilde{D}=\{x \in X: x(t) \in D$ for all $t \in[0, T]\}$. Then there exists $t_{0} \in[0, T]$ such that $\widehat{x}\left(t_{0}\right) \in \partial D$. As $\widehat{x}(t) \equiv p$ for all $t \in[0, T]$, we obtain that $p \in \partial D$. Thus, we have $p \in \partial D$ and $g(p)=0$. It is contradictory to $\left(H_{2}\right)$ because the Brouwer degree $\operatorname{deg}(g, D, 0) \neq 0$.

(ii) When $\widehat{\lambda} \in(0,1]$, as $0=(\mathrm{id}-H)\left(\widehat{x}_{\mathrm{ker}}^{0}, \widehat{x}, \widehat{\lambda}\right)$, we have

$$
\left(\begin{array}{c}
\widehat{x}_{\text {ker }}^{0} \\
\widehat{x}(t)
\end{array}\right)=\left(\begin{array}{c}
\widehat{x}_{\mathrm{ker}}^{0}+(1 / T) \int_{0}^{T} \operatorname{Pf}\left(\tau, \alpha_{\widehat{\lambda}} \circ \widehat{x}(\tau)\right) \mathrm{d} \tau \\
\alpha_{\widehat{\lambda}} \circ \widehat{x}_{\mathrm{ker}}^{0}- \\
-\widehat{\lambda} L_{p}^{-1}(I-P) \int_{0}^{T} f\left(\tau, \alpha_{\widehat{\lambda}} \circ \widehat{x}(\tau)\right) \mathrm{d} \tau \\
+\widehat{\lambda} \int_{0}^{t} f\left(\tau, \alpha_{\widehat{\lambda}} \circ \widehat{x}(\tau)\right) \mathrm{d} \tau
\end{array}\right) .
$$

Thus,

$$
\frac{1}{T} \int_{0}^{T} \operatorname{Pf}\left(\tau, \alpha_{\widehat{\lambda}} \circ \widehat{x}(\tau)\right) \mathrm{d} \tau=0
$$

and

$\widehat{x}(t)=\alpha_{\widehat{\lambda}} \circ \widehat{x}_{\mathrm{ker}}^{0}-\widehat{\lambda} L_{p}^{-1}(I-P) \int_{0}^{T} f\left(\tau, \alpha_{\widehat{\lambda}} \circ \widehat{x}(\tau)\right) \mathrm{d} \tau+\widehat{\lambda} \int_{0}^{t} f\left(\tau, \alpha_{\widehat{\lambda}} \circ \widehat{x}(\tau)\right) \mathrm{d} \tau$.

Noting that:

$$
\begin{aligned}
\left|\frac{\widehat{x}(t)-\widehat{x}(s)}{t-s}\right| & =\frac{1}{|t-s|}\left|\widehat{\lambda} \int_{s}^{t} f\left(\tau, \alpha_{\widehat{\lambda}} \circ \widehat{x}(\tau)\right) \mathrm{d} \tau\right| \\
& \leq \frac{\widehat{\lambda}}{|t-s|} \int_{s}^{t}\left|f\left(\tau, \alpha_{\widehat{\lambda}} \circ \widehat{x}(\tau)\right)\right| \mathrm{d} \tau \leq \widehat{\lambda} M
\end{aligned}
$$

by the definition of $X_{\lambda}$, we obtain $\widehat{x} \in X_{\hat{\lambda}}$, which means that $\alpha_{\hat{\lambda}} \circ \widehat{x}=\widehat{x}$. Now we can rewrite equation (2.12) as

$$
\widehat{x}(t)=\widehat{x}_{\mathrm{ker}}^{0}-\widehat{\lambda} L_{p}^{-1}(I-P) \int_{0}^{T} f(\tau, \widehat{x}(\tau)) \mathrm{d} \tau+\widehat{\lambda} \int_{0}^{t} f(\tau, \widehat{x}(\tau)) \mathrm{d} \tau .
$$

By a similar discussion of equation (2.5), we can prove that $\widehat{x}(t)$ is a solution of equation $(1)_{\widehat{\lambda}}$. By hypothesis $\left(H_{1}\right)$, we know that $\widehat{x}(t) \notin \partial D$ for any $t \in[0, T]$. This is a contradiction to $\widehat{x} \in \partial \widetilde{D}$. 
By (i) and (ii), we obtain that

$$
0 \notin(\mathrm{id}-H)(\partial((D \cap \operatorname{Ker}(I-Q)) \times \widetilde{D}) \times[0,1]) .
$$

Therefore, by the homotopy invariance and the theory of Brouwer degree, we have

$$
\begin{aligned}
& \operatorname{deg}\left(\mathrm{id}-H\left(x_{\mathrm{ker}}^{0}, \cdot, 1\right),(D \cap \operatorname{Ker}(I-Q)) \times \widetilde{D}, 0\right) \\
& =\operatorname{deg}\left(\mathrm{id}-H\left(x_{\mathrm{ker}}^{0}, \cdot, 0\right),(D \cap \operatorname{Ker}(I-Q)) \times \widetilde{D}, 0\right) \\
& =\operatorname{deg}(g, D \cap \operatorname{Ker}(I-Q), 0) \neq 0 .
\end{aligned}
$$

This means that there exists $\widehat{x}_{*} \in \widetilde{D}$, such that

$$
\left(\begin{array}{l}
\widehat{x}_{* \mathrm{ker}}^{0} \\
\widehat{x}_{*}(t)
\end{array}\right)=\widehat{T}\left(\widehat{x}_{* \mathrm{ker}}^{0}, \widehat{x}_{*}(t), 1\right) .
$$

Similar to the proof in (ii), we get $\widehat{x}_{*} \in X_{1}$. Then,

$$
\widehat{T}\left(\widehat{x}_{* k e r}^{0}, \widehat{x}_{*}(t), 1\right)=T\left(\widehat{x}_{* \mathrm{ker}}^{0}, \widehat{x}_{*}(t), 1\right) .
$$

By equations (2.13) and (2.14), we obtain that $\widehat{x}_{*}$ is a fixed point of $T$ in $X$. Thus, $\widehat{x}_{*}(t)$ is a solution of equation $x^{\prime}=f(t, x)$ with boundary value condition $x(T)=Q x(0)$.

(II) If $\operatorname{Ker}(I-Q)=\{0\}$. In this case, $(I-Q)^{-1}$ exists. Then:

$$
x_{0}=-\lambda L_{p}^{-1}(I-P) \int_{0}^{T} f(\tau, x(\tau)) \mathrm{d} \tau .
$$

We no longer need hypothesis $\left(H_{2}\right)$. Consider the homotopy:

$H(x, \lambda)=-\lambda L_{p}^{-1}(I-P) \int_{0}^{T} f\left(\tau, \alpha_{\lambda} \circ x(\tau)\right) \mathrm{d} \tau+\lambda \int_{0}^{t} f\left(\tau, \alpha_{\lambda} \circ x(\tau)\right) \mathrm{d} \tau$.

Similar to the proof of $\operatorname{Ker}(I-Q) \neq\{0\}$, we have $0 \notin(\mathrm{id}-H)(\partial \widetilde{D} \times$ $[0,1])$. Hence,

$$
\operatorname{deg}(\mathrm{id}-H(\cdot, 1), \widetilde{D}, 0)=\operatorname{deg}(\mathrm{id}-H(\cdot, 0), \widetilde{D}, 0)=\operatorname{deg}(\mathrm{id}, \widetilde{D}, 0)=1 .
$$

This means that there exists $\widehat{x}_{*}$, which satisfies $\widehat{x}_{*}(t) \in D$ for all $t \in[0, T]$, such that:

$$
\widehat{x}_{*}(t)=\widehat{x}_{*}(0)+\int_{0}^{t} f\left(\tau, \widehat{x}_{*}(\tau)\right) \mathrm{d} \tau .
$$


Therefore, $\widehat{x}_{*}(t)$ is a solution of equation $x^{\prime}=f(t, x)$ with boundary value condition $x(T)=Q x(0)$.

By Lemma 1.3 and the proofs in (I) and (II), it is easy to see that APS (1.1) has a $(Q, T)$-affine-periodic solution $x_{*}(t)$, which is an extension of $\widehat{x}_{*}(t)$ on $R^{1}$. Since $x_{*}(t)$ is continuous and $x_{*}(t) \in D$ when $t \in[0, T]$, by hypothesis $\left(H_{1}\right)$, we know that $x_{*}(t) \in D$ for all $t$.

3. Proofs of Corollaries 1 and 2. Consider the auxiliary equation,

$$
x^{\prime}=\lambda f(t, x),
$$

where $\lambda \in[0,1]$. Set

$$
\begin{array}{rlrl}
L_{i} & =\sup \left\{\left|V_{i}(x)\right|:|x| \leq M_{i}\right\}, & L & =\sum_{i=0}^{m} L_{i}, \\
D & =\left\{p \in R^{n}: \sum_{i=0}^{m}\left|V_{i}(p)\right|<L+1\right\}, & V(x)=\sum_{i=0}^{m}\left|V_{i}(x)\right| .
\end{array}
$$

By $\left(H_{4}\right)$, we claim that $D$ is bounded for $\lambda \in(0,1]$, and every possible $(Q, T)$-affine-periodic solution $x(t)$ of $(1)_{\lambda}$ satisfies

$$
x(t) \in D \quad \text { for all } t .
$$

In fact, since $x(t)$ is a $(Q, T)$-affine-periodic function and $Q \in O(n)$, there exists a sequence $\left\{t_{j}\right\} \subset R^{1}$ such that

$$
\left|V\left(x\left(t_{j}\right)\right)\right| \longrightarrow \sup _{R^{1}}|V(x(t))|<\infty, \quad \text { as } j \rightarrow \infty .
$$

Hence, for some $i$,

$$
\left|V_{i}\left(x\left(t_{j}\right)\right)\right| \longrightarrow \sup _{R^{1}}\left|V_{i}(x(t))\right|, \quad \text { as } j \rightarrow \infty,
$$

which implies that

$$
\frac{\mathrm{d}}{\mathrm{d} t} V_{i}\left(x\left(t_{j}\right)\right)=\left\langle\nabla V_{i}\left(x\left(t_{j}\right)\right), \lambda f\left(t_{j}, x\left(t_{j}\right)\right)\right\rangle \longrightarrow 0, \quad \text { as } j \rightarrow \infty .
$$

By $\left(H_{3}\right)$, this result yields

$$
\left|x\left(t_{j}\right)\right|<M_{j}, \quad \text { as } j \rightarrow \infty .
$$


Consequently, by the definition of $D$ and equation (3.1), we have

$$
x(t) \in D \quad \text { for all } t .
$$

Thus, hypothesis $\left(H_{1}\right)$ holds.

If $\operatorname{Ker}(I-Q)=\{0\}$, by the proof of Theorem 1.4, we know that equation (1.1) admits a $(Q, T)$-affine-periodic solution.

Now we prove that, if $\operatorname{Ker}(I-Q) \neq\{0\}$,

$$
\operatorname{deg}(g, D \cap \operatorname{Ker}(I-Q), 0) \neq 0 .
$$

Indeed, consider the homotopy,

$H(p, \lambda)=\lambda \operatorname{sgn}\left(\left.\left\langle\nabla V_{0}(\cdot), P f(t, \cdot)\right\rangle\right|_{\partial\left(B_{M_{0}} \cap \operatorname{Ker}(I-Q)\right)}\right) \nabla V_{0}(p)+(1-\lambda) g(p)$, where $(p, \lambda) \in\left(B_{M_{0}} \cap \operatorname{Ker}(I-Q)\right) \times[0,1]$. It follows that

$$
\begin{aligned}
\left\langle\nabla V_{0}(p), H(p, \lambda)\right\rangle= & \lambda \operatorname{sgn}\left(\left.\left\langle\nabla V_{0}(\cdot), P f(t, \cdot)\right\rangle\right|_{\partial\left(B_{M_{0}} \cap \operatorname{Ker}(I-Q)\right)}\right)\left|\nabla V_{0}(p)\right|^{2} \\
& +(1-\lambda)\left\langle\nabla V_{0}(p), g(p)\right\rangle .
\end{aligned}
$$

For any $(p, t) \in \partial\left(B_{M_{0}} \cap \operatorname{Ker}(I-Q)\right) \times R^{1}$, by $\left(H_{3}\right)$ we know that the sign of $\left\langle\nabla V_{0}(p), P f(t, p)\right\rangle$ does not change. And, by the definition of $g(a)$, we have

$$
\begin{aligned}
\left\langle\nabla V_{0}(p), g(p)\right\rangle & =\left\langle\nabla V_{0}(p), \frac{1}{T} \int_{0}^{T} \operatorname{Pf}(s, p) \mathrm{d} s\right\rangle \\
& =\frac{1}{T} \int_{0}^{T}\left\langle\nabla V_{0}(p), \operatorname{Pf}(s, p)\right\rangle \mathrm{d} s .
\end{aligned}
$$

This means that $\left\langle\nabla V_{0}(p), g(p)\right\rangle$ always has the same sign with $\left\langle\nabla V_{0}(p)\right.$, $P f(t, p)\rangle$. Also, by $\left(H_{3}\right)$, we know that $\left|V_{0}(p)\right| \neq 0$ when $p \in \partial\left(B_{M_{0}} \cap\right.$ $\operatorname{Ker}(I-Q))$. Consequently, the right hand side of equation (3.2) is nonzero.

Thus, $\left\langle\nabla V_{0}(p), H(p, \lambda)\right\rangle \neq 0$ for all $(p, \lambda) \in \partial\left(B_{M_{0}} \cap \operatorname{Ker}(I-Q)\right) \times$ $[0,1]$, which implies that $0 \notin H\left(\partial\left(B_{M_{0}} \cap \operatorname{Ker}(I-Q)\right) \times[0,1]\right)$.

The homotopy invariance of the Brouwer degree implies

$$
\begin{aligned}
& \operatorname{deg}\left(g, B_{M_{0}} \cap \operatorname{Ker}(I-Q), 0\right) \\
& =\operatorname{deg}\left(\operatorname{sgn}\left(\left.\left\langle\nabla V_{0}(\cdot), P f(t, \cdot)\right\rangle\right|_{\partial\left(B_{M_{0}} \cap \operatorname{Ker}(I-Q)\right)}\right)\right. \\
& \left.\quad \nabla V_{0}, B_{M_{0}} \cap \operatorname{Ker}(I-Q), 0\right) \neq 0 .
\end{aligned}
$$


Hence, hypothesis $\left(H_{2}\right)$ holds. Thus, Corollary 1.7 follows from Theorem 1.4 .

By Corollary 1.7, we can easily prove Corollary 1.8 as follows.

Proof. Let

$$
V(x)=\frac{1}{2}|x|^{2}
$$

Then

$$
\operatorname{deg}\left(\nabla V, B_{M} \cap \operatorname{Ker}(I-Q), 0\right)=\operatorname{deg}\left(\mathrm{id}, B_{M} \cap \operatorname{Ker}(I-Q), 0\right)=1,
$$

and

$$
V(x) \longrightarrow \infty, \quad \text { as }|x| \rightarrow \infty .
$$

Thus, the conclusion follows from Corollary 1.7.

4. Proof of Theorem 2. In Corollary 1.7, we proved that equation (1.1) admits an affine-periodic solution when hypotheses $\left(H_{3}\right)$ $\left(H_{5}\right)$ hold. Notice that $\left(H_{3}\right)$ requires the properties of $V(x)$ far enough from the origin. For some certain $f(t, x)$, such a $V(x)$ may not be easily constructed. Thus, we give Theorem 1.9 which requires the properties of $V(x)$ on the boundary of the domain. It makes the construction of the Lyapunov function easier in some situations.

Proof. By $\left(H_{7}\right)$ and the definition of $g(a)$, it follows that

$$
g(a) \neq 0 \text { for all } a \in \partial D \text {. }
$$

Consider the homotopy:

$H(a, \lambda)=\lambda \operatorname{sgn}\left(\left.\langle\nabla V(\cdot), P f(t, \cdot)\rangle\right|_{\partial(D \cap \operatorname{Ker}(I-Q))}\right) \nabla V(a)+(1-\lambda) g(a)$, where $(a, \lambda) \in \partial(D \cap \operatorname{Ker}(I-Q)) \times[0,1]$.

A similar argument to the proof of Corollary 1.7, together with $\left(H_{7}\right)$ and $\left(H_{8}\right)$, implies

$$
\operatorname{deg}(g, D \cap \operatorname{Ker}(I-Q), 0) \neq 0 .
$$

In the following, we will prove that, for each $\lambda \in(0,1]$ fixed, every possible $(Q, T)$-affine-periodic solution $x(t)$ of $(1)_{\lambda}$ in $\bar{D}$ satisfies

$$
x(t) \in D \quad \text { for all } t .
$$


If this is false, then there exists a $t_{0} \in R^{1}$ such that

$$
x\left(t_{0}\right) \in \partial D .
$$

By $\left(H_{7}\right)$, there is an $\epsilon_{0}>0$ such that

$$
|\langle\nabla V(x), f(t, x)\rangle| \geq \frac{\sigma}{2},
$$

provided $t \in R^{1}, x \in \bar{D}$ and $\operatorname{dist}(x, \partial D) \leq \epsilon_{0}$. Since $x(t)$ is $(Q, T)$-affine-periodic and $Q \in O(n)$, there exists a $\left\{t_{k}\right\} \subset R^{1}$ with $t_{k} \rightarrow \infty(k \rightarrow \infty)$ such that

$$
\left\langle\nabla V\left(x\left(t_{k}\right)\right), \lambda f\left(t_{k}, x\left(t_{k}\right)\right)\right\rangle=V^{\prime}\left(x\left(t_{k}\right)\right) \longrightarrow 0, \quad k \rightarrow \infty,
$$

which implies that

$$
x\left(t_{k}\right) \in D, \quad \operatorname{dist}\left(x\left(t_{k}\right), \partial D\right)>\epsilon_{0}, \quad k \rightarrow \infty .
$$

On the other hand, there exists a $\left\{\tau_{k}\right\} \subset R^{1}$ with $\tau_{k} \rightarrow \infty(k \rightarrow \infty)$ such that

$$
x\left(\tau_{k}\right) \in D, x\left(\tau_{k}\right) \longrightarrow x\left(t_{0}\right), \quad \tau_{k} \rightarrow \infty .
$$

By the properties of $\left\{x\left(t_{k}\right)\right\},\left\{x\left(\tau_{k}\right)\right\}$ and the continuity of $V^{\prime}(x(t))$, there are $s_{1}, s_{2} \in R^{1}$ such that

$$
\begin{aligned}
& x\left(s_{1}\right) \in D, \operatorname{dist}\left(x\left(s_{1}\right), \partial D\right)<\epsilon_{0} ; \\
& x\left(s_{2}\right) \in D, \operatorname{dist}\left(x\left(s_{2}\right), \partial D\right)<\epsilon_{0} ; \\
& V^{\prime}\left(x\left(s_{1}\right)\right)>0 ; \quad V^{\prime}\left(x\left(s_{2}\right)\right)<0 .
\end{aligned}
$$

However, $\langle\nabla V(x), \lambda f(t, x)\rangle$ keeps a sign on the domain dist $(x, \partial D) \leq$ $\epsilon_{0}$. This is a contradiction.

Now, the conclusion follows from Theorem 1.4.

5. Proof of Theorem 3. Consider the auxiliary equation:

$$
x^{\prime}=\lambda f(t, x),
$$

where $\lambda \in[0,1]$.

For $\epsilon>0$ small enough, define a set $D_{\epsilon}$ as

$$
D_{\epsilon}=\{x \in D: \operatorname{dist}(x, \partial D)>\epsilon\} .
$$

Then $\partial D_{\epsilon}$ is piecewise smooth. Since, for each $(t, p) \in R^{1} \times \partial D$ and $h \in H(f), \lambda h(t, p)$ is inward to $D$, there exists an $\epsilon_{0}>0$ small enough 
such that, for each $(t, p) \in R^{1} \times \partial D_{\epsilon}, h \in H(f)$ and $\epsilon \in\left[0, \epsilon_{0}\right], \lambda h$ is inward to $D_{\epsilon}$. This obviously implies that every possible $(Q, T)$-affineperiodic solution $x_{\lambda}(t)$ of $(1)_{\lambda}$ satisfies that $x_{\lambda}(t) \in D_{\epsilon_{0}}$ for all $t$.

It suffices to count the Brouwer degree $\operatorname{deg}(g, D \cap \operatorname{Ker}(I-Q), 0)$, when $\operatorname{Ker}(I-Q) \neq\{0\}$.

We assume without loss of generality that $g$ satisfies the local Lipschitz condition; otherwise, we use the $C^{1}$ sequence $\left\{g_{k}\right\}$ such that $g_{k} \rightarrow g(k \rightarrow \infty)$ uniformly on $\overline{D \cap \operatorname{Ker}(I-Q)}$, and consider the map $g_{k}$.

Consider the system

$$
y^{\prime}=g(y)
$$

Let $y\left(t, y_{0}\right)$ denote the solution of equation (5.1) with the initial value $y(0)=y_{0}$ for $y_{0} \in \overline{D \cap \operatorname{Ker}(I-Q)}$. By Poincaré's theorem, there exists a homomorphism $\widehat{B}: \overline{D \cap \operatorname{Ker}(I-Q)} \rightarrow B(0,1)$, where $B(0,1) \subset R^{m}$ is the unity ball centered at the origin, $m=\min \{n$, $\operatorname{dim} \operatorname{Ker}(I-Q)\}$. Set the map

$$
J\left(y_{0}, t\right)= \begin{cases}(1 / t)\left[y_{0}-y\left(t, y_{0}\right)\right]=-(1 / t) \int_{0}^{t} g\left(y\left(s, y_{0}\right)\right) d s & t>0 \\ -g\left(y_{0}\right) & t=0 .\end{cases}
$$

Then $J$ is continuous on $\overline{D \cap \operatorname{Ker}(I-Q)} \times[0,1]$. Notice that, for each point $q \in \partial D, f(s, q)$ is inward to $D$ for all $s \in R^{1}$ by $\left(H_{9}\right)$. By the definition in $\left(H_{2}\right)$, we know that $g$ is inward to $D$. Also, $g(a) \in$ $\operatorname{Ker}(I-Q)$ for any $a \in \operatorname{Ker}(I-Q)$. We have $y(t, \cdot): \overline{D \cap \operatorname{Ker}(I-Q)} \rightarrow$ $D \cap \operatorname{Ker}(I-Q)$ for all $t>0$, and $J(\partial(D \cap \operatorname{Ker}(I-Q)) \times[0,1]) \neq 0$, which implies that

$$
\left.\left(\mathrm{id}-\widehat{B}\left(y\left(t, \widehat{B}^{-1}(\cdot)\right)\right)\right)\right|_{S^{m} \times(0,1]} \neq 0,
$$

where $\widehat{B}\left(y\left(t, \widehat{B}^{-1}(\cdot)\right)\right): B(0,1) \rightarrow B(0,1) \backslash S^{m}$ for all $t>0$. Hence, the homotopy invariance implies

$\operatorname{deg}(-g, D \cap \operatorname{Ker}(I-Q), 0)=\operatorname{deg}(-J(\cdot, t), D \cap \operatorname{Ker}(I-Q), 0)$ for all $t>0$, and the Rouché theorem yields:

$$
\operatorname{deg}\left(\mathrm{id}-\widehat{B}\left(y\left(t, \widehat{B}^{-1}(\cdot)\right)\right), B(0,1) \backslash S^{m}, 0\right)=1 \quad \text { for all } t>0 .
$$


Since $\widehat{B}: \overline{D \cap \operatorname{Ker}(I-Q)} \rightarrow B(0,1)$ is a homomorphism, by Leray's formula, we have

$$
\begin{aligned}
& \operatorname{deg}(-J(\cdot, t), D \cap \operatorname{Ker}(I-Q), 0) \\
& \quad= \pm \operatorname{deg}\left(\mathrm{id}-\widehat{B}\left(y\left(t, \widehat{B}^{-1}(\cdot)\right)\right), B(0,1) \backslash S^{m}, 0\right)= \pm 1 \quad \text { for all } t>0 .
\end{aligned}
$$

Consequently,

$$
\operatorname{deg}(-g, D \cap \operatorname{Ker}(I-Q), 0)= \pm 1 \text {. }
$$

Now, the conclusion follows from Theorem 1.4.

Acknowledgments. The authors are grateful to the referee for his/her patience and valuable comments.

\section{REFERENCES}

1. Y. Chen, J.J. Nieto and D. O'Regan, Anti-periodic solutions for evolution equations associated with maximal monotone mappings, Appl. Math. Lett. 24 (2011), 302-307.

2. Y. Chen, D. O'Regan and Ravi P. Agarwal, Anti-periodic solutions for semilinear evolution equations in Banach spaces, Appl. Math. Comp. 38 (2012), $63-70$.

3. N. Dilan and M. Fečkan, On symmetric and periodic solutions of parametric weakly nonlinear ODE with time-reversal symmetries, Bull. Belgian Math. Soc. 18 (2011), 896-923.

4. L.H. Erbe, W. Krawcewicz and J. Wu, Leray-Schauder degree for semilinear Fredholm maps and periodic boundary value problems of neutral equations, Nonlin. Anal. 15 (1990), 747-764.

5. __ A composite coincidence degree with applications to boundary value problems of neutral equations, Trans. Amer. Math. Soc. 335 (1993), 459-478.

6. J.K. Hale and J. Mawhin, Coincidence degree and periodic solutions of neutral equations, J. Diff. Eq. 15 (1974), 295-307.

7. T. Küpper, Y. Li and B. Zhang, Periodic solutions for dissipative-repulsive systems, Tohoku Math. J. 52 (2000), 321-329.

8. Y. Li and F. Huang, Levinson's problem on affine-periodic solutions, Adv. Nonlin. Stud. 15 (2014), 241-252.

9. R. de la Llave, A tutorial on KAM theory, in Pure mathematics on smooth ergodic theory and its applications, American Mathematical Society, Providence, RI, 2001.

10. J. Mawhin and R.E. Gaines, Coincidence degree and nonlinear differential equations, Springer-Verlag, Berlin, 1977.

11. H. Wang, X. Yang and Y. Li, Rotating-symmetric solutions for nonlinear systems with symmetry, Acta Math. Appl. Sin. 31 (2015), 307-312. 
12. J. Wu, H. Xia and B. Zhang, Topological transversality and periodic solutions of neutral functional-differential equations, Proc. Roy. Soc. Edinb. 129 (1999), $199-220$.

13. T. Yoshizawa, Stability theory and the existence of periodic solutions and almost periodic solutions, Springer-Verlag, New York, 1975.

14. Y. Zhang, X. Yang and Y. Li, Affine-periodic solutions for dissipative systems, Abs. Appl. Anal. 2013 (2013), article ID 157140, doi:10.1155/2013/ 157140.

College of Mathematics, Jilin University, Changchun 130012, P.R. China and School of Science, Communication University of China, Beijing 100024, P.R. CHINA

Email address: arima1984@163.com

School of Mathematics and Statistics, Center for Mathematics and Interdisciplinary Sciences, Northeast Normal University, Changchun 130024, P.R. CHINA

Email address: yangxuemath@163.com

School of Mathematics and Statistics, Center for Mathematics and InterDisciplinary Sciences, Northeast Normal University, Changchun 130024 , P.R. China

Email address: liyongmath@163.com 